Learning Objectives: The experimental assessments of the new prostheses (PORP and TORP) in cadaveric temporal measurements provide objective ways to predict their functional outcomes and benefits prior to their clinical application.

The middle-ear in human ear converts and transmits acoustically-induced sound stimuli to the inner ear. The middle-ear structures can be damaged by various middleear pathologies. The damaged middle-ear structures are frequently reconstructed by surgical procedures to rearrange or to replace the impaired middle-ear structures with an implantable prosthesis. Especially, the partial ossicular reconstruction prosthesis (PORP) and total ossicular reconstruction prosthesis (TORP) are used to provide direct connection between the tympanic membrane and the stapes. While such tympanoplasty surgeries are common these days, stable positioning of the prosthesis and reliable connection between the prosthesis and the remaining ossicular structure are still difficult to achieve.

In this study, four newly-introduced prostheses for tympanoplasty were assessed in cadaveric temporal bones; two PORPs with a ball joint and a notch for placement under the malleus and two supplemental devices for TORP, Omega Connector and TotalOption Connector. All the prostheses were implanted to the temporal bones in sequence, and time for implantation was measured for each of the prostheses.

With each of the prostheses implanted, motion of the stapes footplate and the volume displacement at the round window membrane were measured using a laser Doppler vibrometer (LDV).

The measured quantities were assessed as the functional outcomes of the surgical reconstruction with the corresponding prosthesis, in comparison with sound transmission in normal ears Preliminary results indicate that middle-ear reconstructions with the newly-developed prostheses resulted in surgical outcomes comparative to normal middle-ear. Further, they provide relatively easy handling of the prostheses during the surgeries and relatively secure connection between the prostheses and the remaining middle-ear structures and thus relatively small risk of postoperative dislocation compared to current prostheses for tympanoplasty.

doi:10.1017/S0022215116002759

\section{Difficult Situations in Cholesteatoma Surgery (N713)}

\section{ID: 713.1}

Current trends in managing complications of chronic otitis media with cholesteatoma

Presenting Author: Jyoti Dabholkar

Jyoti Dabholkar, Arpit Sharma, Jaini Lodha, Nitish Virmani, Shruti Bansal

\section{King Edward Memorial Hospital}

Learning Objectives: 1. Complications secondary to cholesteatoma still remain a formidable challenge in developing countries. A high index of suspicion is necessary to prevent significant morbidity and mortality. 2. CT scan plays a pivotal role in diagnosis of both intracranial and extracranial complications. 3. While the initial management may differ, canal wall down mastoidectomy remains the most reliable surgical procedure in these patients.

Introduction: Complications secondary to cholesteatoma are associated with significant morbidity and mortality. Despite a significant decline in the incidence of these complications in developed countries, they still pose a considerable challenge in developing countries. The present study has been conducted to outline our experience in managing complications of cholesteatoma.

Materials and Methods: This study was a retrospective review at KEM Hospital, India of clinical charts of patients with cholesteatoma who had presented with clinical or radiological evidence of complications and had undergone surgical interventions between 2008 and 2013. Patient demographics, clinical course, investigations, management and postoperative outcomes were analyzed.

Results: Of the 469 patients that underwent surgery for cholesteatoma, complications were observed in 86 patients $(18.33 \%)$. Intracranial complications included meningitis $1.06 \%$, brain abscess $3.2 \%$, sigmoid sinus thrombophlebitis $1.9 \%$ and subdural empyema $1.06 \%$. Extracranial complications included labyrinthine fistula $4.6 \%$, facial paralysis $2.9 \%$, zygomatic abscess $0.4 \%$, post-auricular abscess $6.39 \%$, neck abscess $1.2 \%$ and labyrinthitis $0.2 \%$. HRCT temporal bone and CT Brain with contrast was done to establish the diagnosis of these complications. With combined neurosurgical intervention for intracranial complications and canal wall down (CWD) mastoidectomy as the definitive procedure, complete eradication of cholesteatoma was achieved.

Conclusions: Complications secondary to cholesteatoma still remain a formidable challenge in developing countries. A high index of suspicion is necessary to prevent significant morbidity and mortality. CT scan plays a pivotal role in diagnosis of both intracranial and extracranial complications. While the initial management may differ, canal wall down mastoidectomy remains the most reliable surgical procedure in these patients.

doi: $10.1017 / \mathrm{S} 0022215116002760$

\section{Difficult Situations in Cholesteatoma Surgery (N713)}

\section{ID: 713.2}

The Evolution of Bone Anchored Hearing Aids (BAHA) in the Indian Subcontinent

\author{
Presenting Author: Sunil Narayan Dutt \\ Sunil Narayan Dutt ${ }^{1}$, Apurv Kumar ${ }^{2}$ \\ ${ }^{1}$ Apollo International Hospitals Group, \\ ${ }^{2}$ Ashadeep ENT Centre, Chief Audiologist
}

Learning Objectives: 1. to understand the prevalence and incidence of partial deafness and the various indications for candidacy for BAHA in India 2. to comprehend issues 
related to awareness, training of professionals and the deterrents for developing a hearing implant technology such as BAHA in a developing country such as India.

Introduction: The introduction of hearing implants in the Indian subcontinent started around the late $1980 \mathrm{~s}$ in Mumbai. Many cochlear implant (CI) companies worked towards establishing comprehensive CI centres in India in the 90 s and to date, more than $120 \mathrm{CI}$ centres are established in the subcontinent offering hearing implants to its patients. While CI work has made good strides across the country (nearly 30,000 implants in 25 years is the estimate), other surgically implantable hearing devices including the BAHA have taken time to find application.

Material and Methods: The databases of all CI centres in India that offer BAHA to their patients were reviewed retrospectively. The general databases that were maintained by mentor surgeons that supported BAHA surgeries across the continent were referred to. CI and BAHA surgeons were interviewed regarding candidacy awareness, surgery, postoperative issues and any cost related deterrents.

Results: Of the 120 centres offering cochlear implants to patients, only about 40 have performed BAHA (Cochlear BAHA) surgeries in the past decade with or without mentor surgeons. A total of 248 BAHA implants have been performed including about 26 BAHA Attract surgeries. About 30 children are using BAHA processors on soft bands awaiting BAHA surgery (when they are five years of age). Fixture failures in the paedistric population is about $6 \%$ while wound related skin/soft tissue reactions have occured in $20 \%$ of patients (Holger grades 1 and 2) and $8 \%$ of patients (Holger grades 3 and 4). Longer abutments have been used to address some of the soft tissue hypertrophy issues in about 8 patients. The conventional technique of skin graft and generous soft tissue reductions (about $60 \%$ of the cohort) saw more soft tissue issues compared to the linear incision and minimal soft tissue reduction technique. Personal hygiene issues, tropical climate and scarring properties are perhaps some reasons for a higher rate of soft tissue reactions. More recently, with the advent of the transcutaneous BAHA 4 Attract systems, there have been no healing related issues thus far (26 patients).

Discussion: The impact of partial deafness that would make the majority of candidacy for BAHA is much less compared to profound deafness (bilateral). Awareness regarding bone conduction implants $(\mathrm{BCI})$ despite a number of educational activities across the country leaves a lot to be desired amongst not just the potential candidates (and parents) but also hearing healthcare professionals. Many families of children that are candidates (bilateral microtia, for example) from the semiurban and rural population, are unwilling to go the extra mile to collect the funding for what is perceived as a minor handicap. Cost is most definitely a deterrent and there are no Government schemes that have included BAHA in their coverage list of devices, while there are atleast eight states in the country that have a state funded cochlear implant programme. With increasing awareness, reductions in the costs and the development of the transcutaneous bone conduction devices, it is envisaged that this form of surgical hearing re/habilitation would have a better acceptance and penetration in the subcontinent in the years to come.

doi:10.1017/S0022215116002772

\section{Difficult Situations in Cholesteatoma Surgery (N713)}

\section{ID: 713.3}

Facial Nerve in Cholesteatoma Surgery-

Handling damage and avoiding injury

Presenting Author: Manoj M P

M P Manoj

Mesiarc

Learning Objectives: To help the evolving surgeon to handle the facial nerve with confidence in extensive cholesteatoma, tips to preserve function and methods of handling injury.

Introduction: The facial nerve passes through the middle ear in its bony canal that is sometimes eroded in cholesteatoma, exposing the nerve trunk to injury during instrumentation. There are a few surgical tips to avoid injury and to repair after injury has happened.

Methods: In MESIARC, a tertiary otologic center, various cases of facial nerve palsy secondary to cholesteatoma, or attempted cholesteatoma surgery are handled. By careful understanding of anatomy,use of good magnification, proper instrumentation and meticulous care, we have been able to preserve facial nerve in most of the cases where it has been affected by disease or surgery. In rare cases where this could not be done, a variety of techniques have been used to correct the cosmetic effect of facial paralysis

Results: We have had 18 cases of facial nerve palsy secondary to cholesteatoma extension, six cases of surgical damage to the facial nerve during cholesteatoma surgery. Most of the cases of primary facial palsy due to disease were decompressed with near total recovery of function. Of the post surgical injury, two were managed with cable grafting, one with cross facial anastamosis, one with temporalis swing and the rest were decompressed with reasonable return to function.

Conclusions: A structured approach to the facial nerve helped with radiologic planning is of paramount importance in preservation of facial nerve function after injury either due to disease or previous surgery. A variety of techniques must be available in our armamentarium as no two patients are the same.

Learning Objectives: This presentation gives important tips to assess the facial nerve from a three dimensional view point, study of radiology of the facial nerve and the array of techniques at our disposal for preservation and repair. 